\title{
Pressure Measurements Under Pressure: Simplifying the Diagnosis of Clinically Significant Portal Hypertension
}

\author{
Carlos Benítez ${ }^{1}$
}

Accepted: 6 October 2021 / Published online: 5 November 2021

(c) The Author(s), under exclusive licence to Springer Science+Business Media, LLC, part of Springer Nature 2021

\author{
Abbreviations \\ CSPH Clinically significant portal hypertension. \\ HVPG Hepatic venous pressure gradient \\ cACLD Compensated advanced chronic liver disease \\ AUC Area under the curve \\ LSM Liver stiffness measurement \\ LSPS Liver stiffness to the spleen/platelet score \\ VNT Varices needing treatment
}

Clinically significant portal hypertension (CSPH) is defined as a hepatic venous pressure gradient (HVPG) $\geq 10 \mathrm{mmHg}$. It has high clinical importance as long as it is associated with the development of esophageal varices and hemorrhage, hepatic decompensation (including hepatic encephalopathy and development of ascites), a reduced tolerance to liver resection (as treatment for hepatocellular carcinoma), and death. [1] In cases of compensated cirrhosis and in the absence of portosystemic collaterals, its diagnosis is difficult if dependent on the measurement of the HVPG, which although an essential tool, requires a trained team and is relatively expensive, invasive, and poorly available in most centers. [2]

To address this problem, efforts were made to develop noninvasive tests to facilitate the diagnosis of CSPH. The ANTICIPATE study showed that the liver stiffness to the spleen/platelet score (LSPS) had superior test characteristics (AUC 0.88). In fact, a value 2.65 or higher is associated with a CSPH risk of $80 \%$. Nevertheless, as with the other tests in this study, it fails to discriminate a population with a very low probability of CSPH in compensated advanced chronic liver disease (cACLD). These findings suggest that although there are still several patients with $\mathrm{CACLD}$ that will require

Carlos Benítez

cbenitez@uc.cl

1 Liver Transplant Unit, Gastroenterology Department, Hospital Clínico de la Pontificia Universidad Católica de Chile, Diagonal Paraguay 362, 8330077 Santiago, Chile
HVPG measurement to diagnose CSPH, a population with a high risk can be identified noninvasively. [3]

The findings of the ANTICIPATE study have become even more important after the PREDESCI study was recently published. [4] It enrolled patients with compensated cirrhosis without varices needing treatment (VNT). The authors randomized patients with CSPH (diagnosed with HVPG measurement) to nonselective $\beta$ blockers (propranolol or carvedilol) or placebo with the primary outcome measures of decompensation or death. Notably, the primary outcome occurred in $16 \%$ of patients in the $\beta$ blockers group and in the $27 \%$ of placebo group patients (HR $0.51, p=0.041$ ). This difference was explained by the reduced incidence of ascites in the $\beta$ blockers group. Thus, CSPH in the absence of VNT has become an entity needing treatment, reinforcing the importance of its diagnosis.

More recently, Pons et al. intended to validate the tools generated by the ANTICIPATE study. [5] A positive predictive value of $90 \%$ was considered appropriate to validate a liver stiffness cutoff value in order to confirm CSPH and a negative predictive value of $90 \%$ was considered to definitively exclude CSPH. All patients had HVPG measurements. A total of 836 patients were included. A liver stiffness measurement (LSM) of $\geq 25 \mathrm{kPa}$ was considered the optimal cutoff to confirm CSPH. Nonetheless, in obese NASH patients, the positive predictive value of this threshold was only $62.8 \%$. The authors generated a model incorporating body mass index, LSM, and platelet count to correctly estimate the probability of CSPH in these patients. On the other hand, $\mathrm{LSM} \leq 15 \mathrm{kPa}$ plus platelet count $>150 \times 10^{9} / \mathrm{L}$ was able to exclude CSPH in most of the cases. Based on these results, patients can be non-invasively risk-stratified for CSPH for the purpose of selecting candidates that will benefit from treatment with nonselective $\beta$ blockers, reducing the need for HVPG measurement.

In this issue of Digestive Diseases and Sciences, Podrug et al. attempted to validate the results obtained by Pons et al. in a Croatian population. [6] Validation in different populations is an important aim considering that not every tool has 
a similar diagnostic yield in all patients even when similar patients are tested. Thus, the authors tested the criteria to use $\mathrm{LSM} \geq 25 \mathrm{kPa}$ to confirm and $\mathrm{LSM} \leq 15 \mathrm{kPa}$ plus platelet count $>150 \times 10^{9} / \mathrm{L}$ to exclude $\mathrm{CSPH}$.

Seventy-six compensated patients without portosystemic collaterals (including gastroesophageal varices) were included. The principal etiologies were chronic hepatitis $\mathrm{C}$, alcoholic liver disease, and NASH, with $30.3 \%$ of the patients obese. The diagnosis of cACLD was performed based on a LSM $>10 \mathrm{kPa}$ or liver imaging suggesting liver fibrosis. This is an important issue considering that only 2/11 patients diagnosed with cACLD solely based on liver imaging (although with $\mathrm{LSM}<10 \mathrm{kPa}$ ) had a liver biopsy revealing liver fibrosis. Since the diagnostic yield of ultrasonography is operator dependent, the use of a criterion based on liver stiffness may be preferable.

Although the diagnostic yield of the criterion to confirm CSPH (LSM $\geq 25 \mathrm{kPa}$ ) was a little lower that in Pons' study, it still seems useful, with a specificity $=88.9 \%$ and a positive predictive value $=87.1 \%$. On the other hand, the criteria employed to exclude CSPH (LSM $\leq 15 \mathrm{kPa}$ plus platelet count $>150 \times 10^{9} / \mathrm{L}$ ) had a $100 \%$ of sensitivity and negative predictive value.

Notably, just as was observed in Pons' study, the prevalence of CSPH was lower in patients with NAFLD compared with other etiologies, reducing the usefulness of the employed criteria. Thus, a lower platelet count and a higher liver stiffness were necessary to diagnose CSPH in those patients with elevated body mass index (BMI). Nonetheless, in patients with NAFLD and normal BMI the criteria performed comparably to the entire cohort.

Since CSPH is a significant clinical condition with prognostic implications even in absence of gastroesophageal varices, its diagnosis must be relatively easy to make. Notably, in Podrug's study, $69.7 \%$ of patients fitted the test criteria, a significant finding considering that only $30 \%$ of patients that previously needed HVPG measurement to diagnose CSPH would need a hemodynamic study based on the proposed criteria, reducing the costs involved and simplifying the diagnosis of CSPH.
The accurate diagnosis of CSPH identifies a group of patients that will benefit from nonselective $\beta$ blockers. The criteria tested by Podrug et al. facilitates the identification of a group of patients with a very low probability of having $\mathrm{CSPH}$, while also identifying a group with a high probability of having CSPH. Although the positive predictive value was $87.1 \%$, the physician and the patient can consider the pros and cons of initiating nonselective $\beta$ blockers based on specific considerations. Consequently, a significant number of HVPG measurements can be reasonably avoided, reducing cost, adverse effects and diagnosing CSPH in a significant number of patients that probably would not have access to HVPG measurement.

One of the main shortcomings of Podrug's study is the low number of patients included. Furthermore, since among patients with $\mathrm{LSM} \leq 10 \mathrm{kPa}$, only $2 / 11$ had advanced fibrosis; the diagnosis of cACLD based solely on liver imaging appears to be inaccurate.

In summary, Podrug's study seems valuable adding information about the validation of the criteria proposed by Pons et al. and confirming its findings in a separate population. Considering the accumulated information, it seems reasonable to assume that in a specific population, the diagnosis of CSPH can be accomplished non-invasively in most of cases (Fig. 1) reducing the need of HVPG measurement, providing an indication for treatment with nonselective $\beta$ blockers, improving the prognosis of a significant number of patients.

\section{Take-Home Messages}

1. Among patients with $\mathrm{CACLD}$, a high-risk population of having CSPH can be identified noninvasively employing liver stiffness measurement (LSM).

2. This determination identifies patients likely to benefit from therapy with nonselective $\beta$ blockers, reducing the probability of death and decompensation.

3. The use of LSM and platelet count identifies a population at very low risk for CSPH.

4. The measurement of HVPG is limited to those in whom the diagnosis of CSPH is indeterminate according to the liver stiffness/platelet criteria.

\section{Declarations}

Conflict of interest Nothing to disclose. 


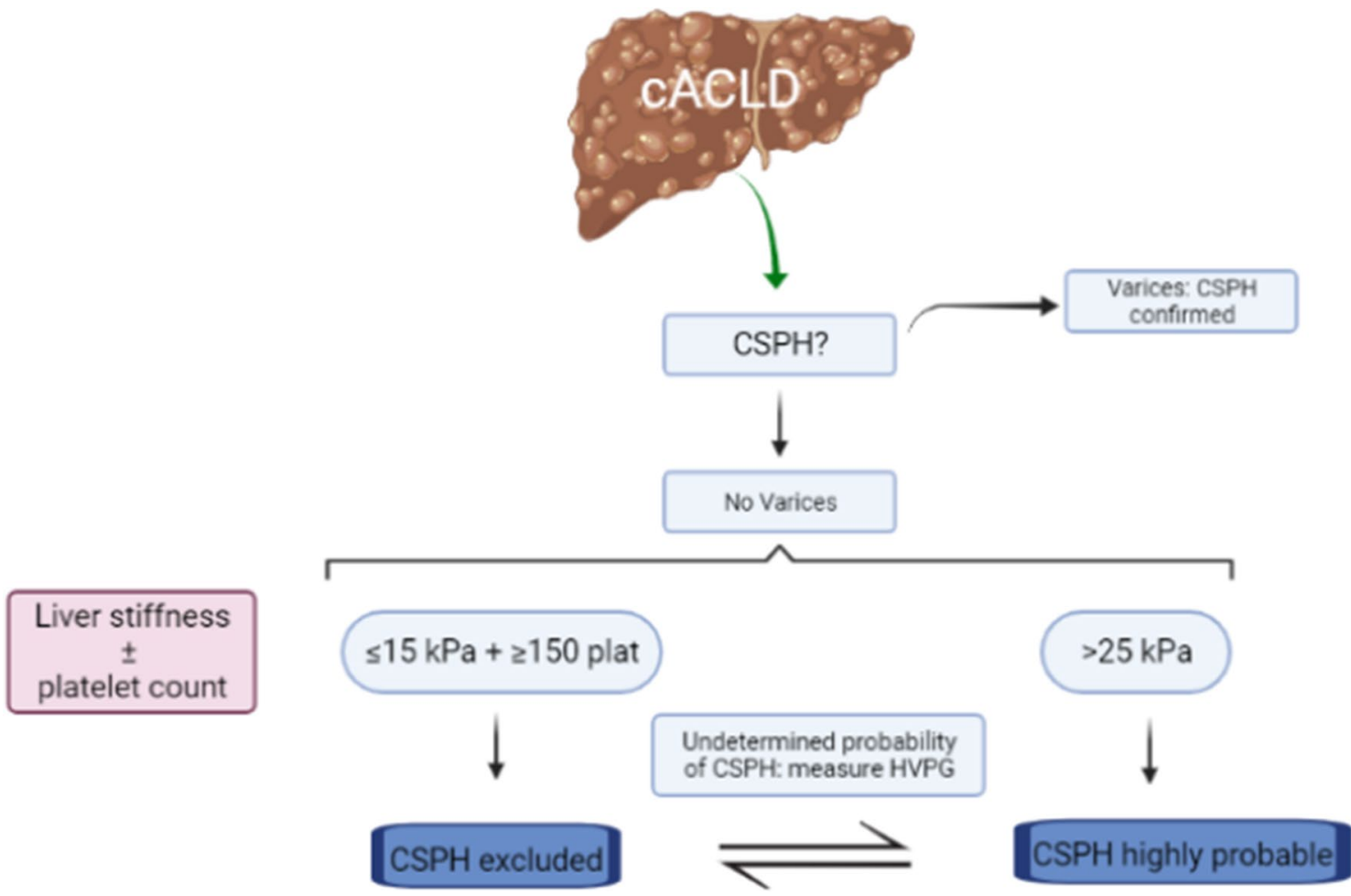

Fig. 1 Algorithm for the diagnosis of CSPH. This strategy allows to diagnose CSPH in a non-invasive manner in most of cases reducing the need of HVPG measurement. Since the described cutoff of liver

\section{References}

1. Vuille-Lessard E, Rodrigues S, Berzigotti A. Noninvasive detection of clinically significant portal hypertension in compensated advanced chronic liver disease. Clin Liver Dis. 2021;25:253-289.

2. Abraldes J, Sarlieve P, Tandon P. Measurement of portal pressure. Clin Liver Dis. 2014;18:779-7792.

3. Abraldes J, Bureau C, Stefanescu H, Augustin S, Ney M, Blasco $\mathrm{H}$ et al. Noninvasive tools and risk of clinically significant portal hypertension and varices in compensated cirrhosis: The "Anticipate" study. Hepatology 2016;64:2173-2184.

4. Villanueva C, Albillos A, Genesca J, García-Pagan JC, Calleja $\mathrm{L}$, Aracil $\mathrm{C}$ et al. $\beta$ blockers to prevent decompensation of cirrhosis in patients with clinically significant portal hypertension (PREDESCI): a randomized, double-blind, placebo controlled, multicentre trial. Lancet 2019;393:1597-1608. stiffness and platelet count are not appropriate to diagnose CSPH in obese patients with NAFLD, this algorithm should not be employed in obese patients diagnosed with NASH

5. Pons M, Augustin S, Scheiner B, Guillaume M, Rosselli M, Rodrigues $\mathrm{S}$ et al. Noninvasive diagnosis of portal hypertension in patients with compensated advanced chronic liver disease. Am J Gastroenterol 2021;116:723-732.

6. Podrug K, Trkulja V, Zelenika M, Bokun T, Madir A, Kanizaj $\mathrm{T}$, et al. Validation of the new diagnostic criteria for clinically significant portal hypertension by platelets and elastography. Dig Dis Sci. (Epub ahead of print). https://doi.org/10.1007/ s10620-021-07277-8.

Publisher's Note Springer Nature remains neutral with regard to jurisdictional claims in published maps and institutional affiliations. 